\title{
Study of Thin-Film Ferroelectric Heterostructures by TEM and PFM
}

\author{
J.R. Jokisaari, ${ }^{*}$ C.T. Nelson, ${ }^{*}$ S.J. Kim, ${ }^{*}$ P. Gao*, S.H. Baek**, C.B. Eom**, and X.Q. Pan * \\ * Department of Materials Science and Engineering, University of Michigan, Ann Arbor, MI 48109 \\ **Department of Materials Science and Engineering, University of Wisconsin-Madison, Madison, \\ WI 53706
}

Ferroelectrics are of significant interest for a new generation of electronic devices such as nonvolatile memories. Transmission electron microscopy (TEM) and scanning probe microscopy (SPM) techniques, notably piezoresponse force microscopy (PFM), have emerged as important methods of characterization for ferroelectric domain structures. A particular emphasis has recently been placed on characterizing time-resolved ferroelectric switching, as this process underlies many potential ferroelectric applications. Each of these has comparative advantages and here we utilize both to characterize ferroelectric thin films of several perovskites: $\mathrm{BaTiO}_{3}$ (BTO), $\mathrm{PbZr}_{0.2} \mathrm{Ti}_{0.8} \mathrm{O}_{3}$ (PZT), and $\mathrm{BiFeO}_{3}$ (BFO) and to corroborate these observations to give a more complete picture of the domain structure and electrical switching mechanisms.

The films were grown by RF sputtering and molecular beam epitaxy onto (001) $\mathrm{SrTiO}_{3}$ or (110) $\mathrm{TbScO}_{3}$. For electrical switching, capacitor type structures were produced by the addition of an epitaxial $\mathrm{SrRuO}_{3}$ or $\mathrm{La}_{0.7} \mathrm{Sr}_{0.3} \mathrm{MnO}_{3}$ electrode. The films were studied using the JEOL JEM-2010F, 3011, and aberration-corrected 2100F, the TEAM0.5 FEI Titan (TEM), the Bruker Dimension Icon AFM, and the Asylum Research MFP-3D AFM.

Switching of ferroelectric domains was studied by grounding the epitaxial electrode and applying a bias using a surface probe. Fig. 1 shows the localized $71^{\circ}$ out-of-plane switching in a $100 \mathrm{~nm}(001) \mathrm{p}$ ( $p$ represents pseudocubic indices) $\mathrm{BiFeO}_{3}$ film. Domain size was a function of the probe size, applied voltage, and switching time as shown the PFM phase image of a grid of switched regions with varying voltage pulses (Fig 1a). The circular blue areas are the switched domains. There is considerable regional variation in the final domain size and shape in the image, however, it qualitatively matches switched regions formed in TEM cross sections (Fig. 1b).

To give a more accurate comparison, and more importantly to gain extra information from a combinatorial approach, we must measure the same location with both techniques. Comparative low

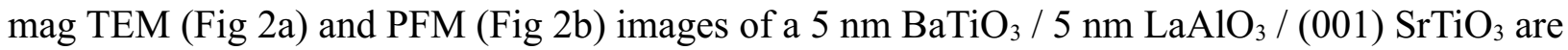
shown of a TEM cross section. Fig. 2c-e shows high magnification TEM of the epitaxial films, VPFM phase overlain on the topography, and the 2-d image of the in-plane phase respectively. A strong response in both vertical and lateral PFM signals can be seen for the ferroelectric films. In Fig. 2e, the lateral phase image, is is possible to distinguish the $\mathrm{LaAlO}_{3}$ and $\mathrm{BaTiO}_{3}$ layers.

In conclusion, by combining PFM and TEM one can fully characterize the polarization of a sample, map the surface topography and characterize detailed spatial information such as interface and defect structures. A comparison of switched regions of a BFO film give good qualitative agreement, suggesting the TEM sample surface does not fundamentally alter the switching process.

This work was supported through NSF grant DMR-0907191, DMR-0820404, and DMR 0723032. 
a

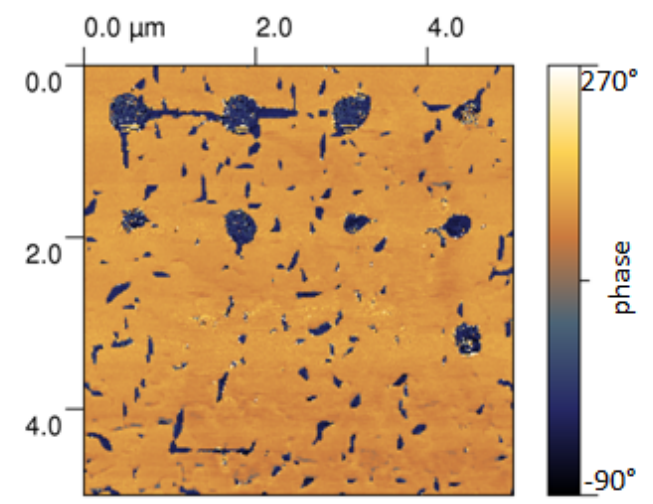

b

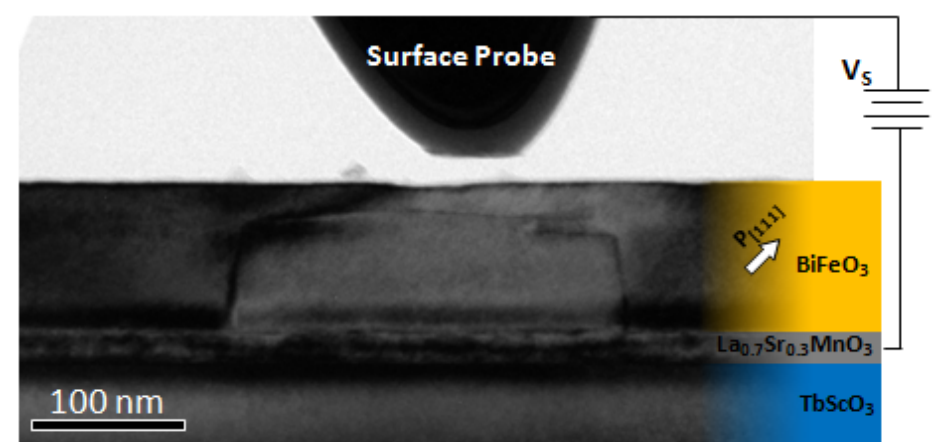

FIG 1. VPFM phase image of a $100 \mathrm{~nm} \mathrm{BiFeO}_{3}$ film with a matrix of switched regions. A 4x4 matrix of spots were switched at $11-44 \mathrm{~V}$ for a $2 \mathrm{~s}$ dwell. The smaller angular regions are defectpinned domain walls. b) TEM image showing the formation of ferroelectric domain in the same film electrically switched in-situ in TEM.

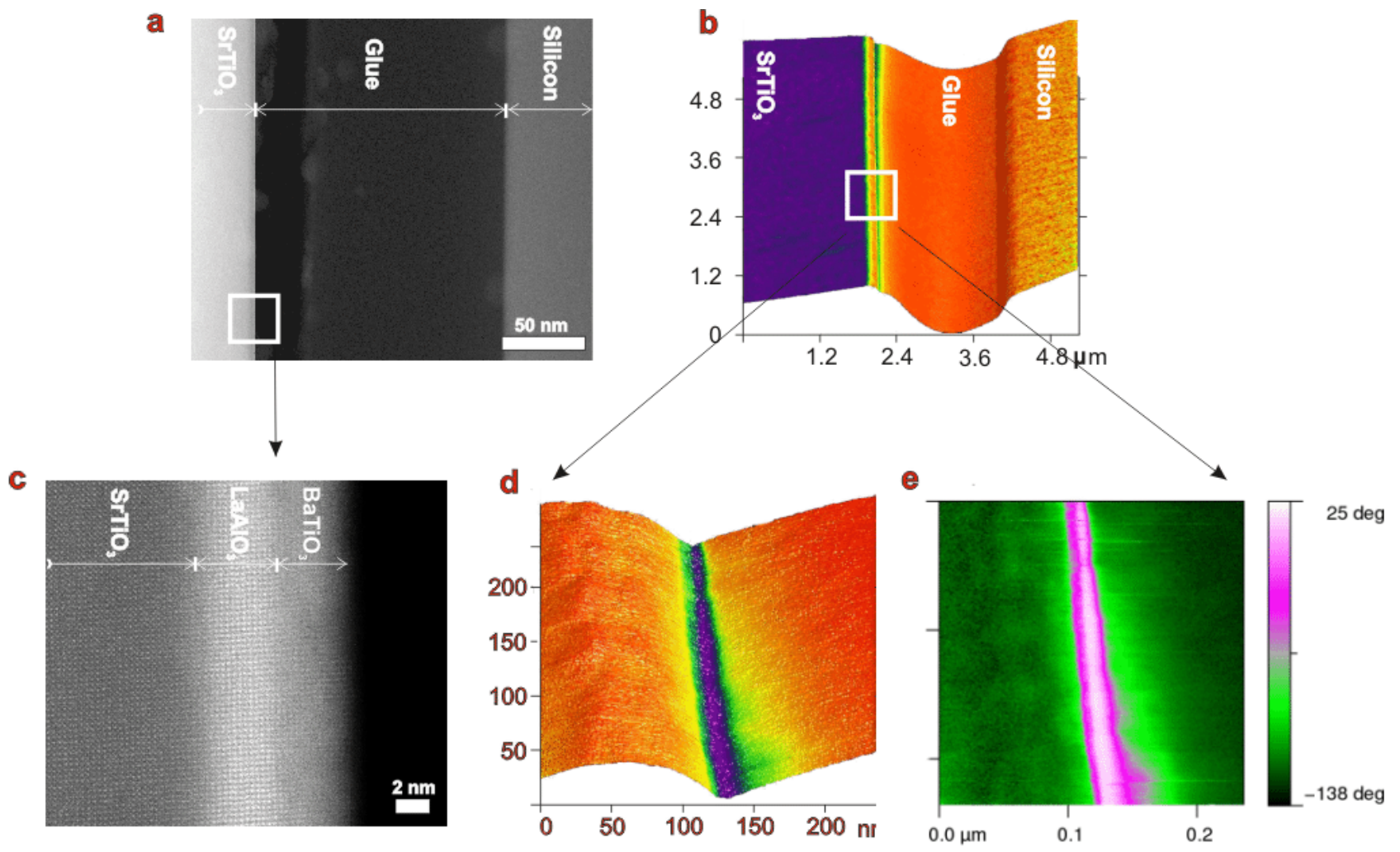

FIG 2. A Low mag image of a TEM cross section with a $5 \mathrm{~nm} \mathrm{BaTiO} / 5 \mathrm{~nm} \mathrm{LaAlO}_{3}$ film stack (a) V-PFM phase image of adjacent area (b). High mag images of the film region in TEM (c), close up V-PFM phase image of the interface (d), and L-PFM phase of the same showing contrast between LAO and BTO (e). 